\title{
PERTINENCIA DE LA FORMACIÓN: OPINIONES DE LAS PERSONAS EGRESADAS DE LA ESCUELA DE QUÍMICA
}

RELEVANCE OF THE EDUCATION: OPINIONS OF PEOPLE GRADUATED FROM THE SCHOOL OF CHEMISTRY

\author{
Volumen 16, Número 2 \\ Mayo-Agosto \\ pp. 1-24
}

Javier Quesada Espinoza

Revista indizada en REDALYC, SCIELO

Revista distribuida en las bases de datos:

LATINDEX, DOAJ, E-REVIST@S, IRESIE, CLASE, DIALNET, SHERPA/ROMEO, QUALIS, MIAR

Revista registrada en los directorios:

ULRICH'S, REDIE, RINACE, OEI, MAESTROTECA, PREAL, $\underline{\text { CLACSO }}$ 


\title{
PERTINENCIA DE LA FORMACIÓN: OPINIONES DE LAS PERSONAS EGRESADAS DE LA ESCUELA DE QUÍMICA \\ RELEVANCE OF THE EDUCATION: OPINIONS OF PEOPLE GRADUATED FROM THE SCHOOL OF CHEMISTRY
}

\begin{abstract}
Javier Quesada Espinoza ${ }^{1}$
Resumen: El presente artículo muestra la valoración de la pertinencia de la formación profesional de personas egresadas de Química. Se valoró la percepción de graduados entre el 2010 y 2014 de la carrera de Bachillerato y Licenciatura en Química de la Universidad de Costa Rica, mediante la aplicación de una encuesta donde se evaluó la pertinencia de bloques temáticos durante la formación, conveniencia de temas presentes y ausentes en la malla curricular actual y el papel de los cursos de Química Industrial, en especial la práctica profesional. Como resultados, se obtuvo información que muestra una desvinculación entre la formación brindada en la universidad y las necesidades del futuro profesional. Se identifican bloques temáticos que no aportan lo esperado. Se manifiestan debilidades en temas asociados a habilidades blandas y tópicos específicos del desarrollo profesional de la Química en el país; dichos temas están vinculados a la gestión, el manejo de personal y el trabajo colaborativo. Se concluye que es necesaria la revisión profunda de la malla curricular con el apoyo de empleadores y personas egresadas para alinearla con las necesidades de la sociedad y promover estrategias didácticas que permitan desarrollar mejor habilidades blandas a lo largo de toda la carrera.
\end{abstract}

Palabras clave: FORMACIÓN PROFESIONAL, QUÍMICA, EDUCACIÓN SUPERIOR, EGRESADOS, HABILIDADES.

Abstract: This paper shows the assessment of the relevance of vocational training of graduates of Chemistry. The perception of graduates between 2010 and 2014 of the career of Bachelor in Chemistry at University of Costa Rica was assessed by applying a survey where the relevance of thematic blocks during vocational training, convenience of present and absent themes in the current curriculum was evaluated and the role of Industrial Chemistry courses, especially professional practice. As a result, information shows a disconnect between the training offered at university and professional future needs was obtained. Thematic blocks that do not provide as expected were identified. Weaknesses are manifested in issues associated with soft skills and specific Chemistry professional development topics in the country; these issues are linked to general management, human resource management and collaborative work. It is concluded that is necessary the thorough review of the curriculum with the support of employers and graduates to align with the needs of society and promote educational strategies to develop better soft skills in the whole career.

Key words: RELEVANCE, PROFESSIONAL, CHEMISTRY, EDUCATION, SKILLS.

${ }_{1}$ Profesor de la Escuela de Química de la Universidad de Costa Rica.

Dirección electrónica: francisco.quesada@ucr.ac.cr

Artículo recibido: 14 de mayo, 2015

Enviado a corrección: 28 de setiembre, 2015

Aprobado: 18 de enero, 2016 


\section{Introducción}

A partir del año 2007 entró en vigencia la última modificación sustancial a la malla curricular de la carrera de Bachillerato y Licenciatura en Química de la Universidad de Costa Rica. Las primeras personas graduadas que cumplieron con las modificaciones que se realizaron, empezaron a graduarse como bachilleres en el año 2010. El espíritu de estos cambios, esencialmente, era brindar al estudiantado la oportunidad de escoger cursos optativos según sus necesidades y preferencias, además de favorecer su contacto con la realidad profesional por medio de la implementación de una práctica profesional. Para lograr esto se redujo el número de cursos obligatorios en el área de la Química inorgánica, la Química orgánica y la Química analítica. La malla curricular actual se puede resumir en los bloques temáticos que se detallan en el Cuadro 1.

\section{Cuadro 1}

Síntesis de la malla curricular de la carrera de Química dividida por grupos temáticos

\begin{tabular}{|l|c|}
\hline \multicolumn{1}{|c|}{ Grupos temáticos } & Número de cursos* \\
\hline Formación humanística & Seis \\
\hline Matemática & Tres \\
\hline Biología & Dos \\
\hline Física & Tres \\
\hline Química general & Cuatro \\
\hline Química analítica & Cuatro \\
\hline Química orgánica & Cuatro \\
\hline Físico-Química & Cuatro \\
\hline Química inorgánica & Tres \\
\hline Química industrial & Cuatro \\
\hline Seminarios de sección & Dos \\
\hline Optativas relativas a Química & Tres \\
\hline
\end{tabular}

* Todos los cursos de Química, física y biología tienen paralelamente uno de laboratorio ya contabilizado en este cuadro, con excepción de un curso de Química inorgánica.

Fuente: Elaboración propia.

La práctica profesional fue el cambio más importante, pues es una nueva estrategia didáctica que no había sido explorada en la Escuela de Química. Por eso, es necesario valorar cómo está funcionando, y si está generando y reforzando las competencias necesarias para el futuro profesional.

Existen muchas opiniones encontradas sobre los resultados de este cambio. Evidentemente, cada una depende de la posición que tenga el consultado en todo este 
proceso: estudiante, docente o empleador o empleadora. Sin embargo, la mayoría son percepciones no sistematizadas como para establecer una conclusión al respecto.

Dentro del proceso para la acreditación de la carrera, se llevó a cabo en el 2011 la recopilación de información referente a la idoneidad de la presente malla curricular. La misma mostró debilidades que generaron algún grado de preocupación y que también fueron apuntadas por evaluadores y evaluados externos. Como consecuencia, en el proceso de reacreditación es una de las líneas de trabajo en la actualidad.

La estructuración adecuada del currículum de una carrera no puede ser dictada por solo uno de los integrantes (estudiantado, docentes o personas empleadoras). Es fundamental que todos los involucrados aporten para lograr desarrollar profesionales más acordes a las necesidades del mercado laboral y, por ende, a la sociedad a la que pertenecen como individuos. Aquí surge, como fundamental, la opinión de las personas interesadas directas, el estudiantado que ha egresado. Se trata de una población que conoce la realidad académica (porque la vivió) y está inmersa en la laboral y puede opinar, presumiblemente, con buen criterio.

Con el fin de conocer la percepción de quienes se han egresado desde el 2010, se realizó una investigación, por medio de una encuesta a graduadas y graduados de bachillerato en Química, para tratar de establecer la pertinencia de la malla curricular para el ejercicio de la profesión. El objetivo fue definir si los bloques de cursos por área o sub-área del conocimiento son los necesarios. Igualmente se buscó establecer la utilidad de las asignaturas brindadas por la Sección de Química Industrial de la Escuela de Química, en especial la correspondiente al desarrollo de la práctica profesional.

El énfasis en esta sección se debe a que es la llamada a brindar el puente entre la realidad académica y profesional, que debe facilitar la incorporación de quienes se egresan al mercado laboral. En las siguientes páginas se describirán los principales hallazgos emanados de este estudio.

\section{Referente teórico}

En los últimos 20 años se ha dado un cambio en la forma en que se conceptualiza la formación para preparar profesionales en cualquier área. Esta nueva estructura se inicia en Europa con la Declaración de Bolonia, en la cual la Unión Europea establece un acuerdo para crear un Espacio Europeo de Educación Superior (EEES), con el que se buscaba el mutuo reconocimiento de títulos, facilitar la movilidad entre los países, aumentar la 
competitividad, entre otros aspectos (Ministros Europeos de Educación, 1999). La manera que ha encontrado el sistema de educación europeo para lograr una formación equivalente es por medio de la definición de competencias que debe cumplir una persona para desempeñarse bien en una actividad determinada. Esto ha dado un giro importante a la educación superior en Europa, e incluso está penetrando la educación básica.

Lo primero que es importante definir es el término competencia. Como menciona Posada Álvarez (2001), el concepto de competencia es diverso, depende del enfoque o perspectiva que se le quiera dar; sin embargo, el más generalizado es el de saber hacer en un contexto. Al respecto, Díaz Barriga y Rigo (citado por Irigoyen, Jiménez y Acuña, 2011, p.247), presentan la siguiente definición para competencias:

Se trata de una capacidad para resolver problemas que se aplica de manera flexible y pertinente, adaptándose al contexto y a las demandas que plantean situaciones diversas (...) desde la óptica de los promotores de la $\mathrm{EBC}^{3}$, la competencia no se limita a los aspectos procedimentales del conocimiento, a la mera posesión de habilidades y destrezas, sino que se ve acompañada necesariamente de elementos teóricos y actitudinales.

La competencia debe estar conformada por tres saberes esenciales: saber conocer, saber hacer y saber ser. El primero está relacionado con los conocimientos necesarios para desempeñarse idóneamente en la labor a ejecutar. Estos pueden ser muy complejos o bastantes básicos, todo dependerá de la actividad a desarrollar. Por otro lado se tiene el saber hacer, el que implica la aplicación en un contexto real de lo aprendido. Muy importante en labores que involucran mucho trabajo con las manos, diseño o elaboración de algo. Finalmente el saber ser, ligado a la capacidad de la persona para evaluar como aportar y a ser crítico con la actividad que se ejecuta (Tobón, 2005).

Quizás la última definición descrita engloba mejor lo que debe ser una competencia debidamente desarrollada. Dado que existen muchas competencias importantes para el desempeño de las múltiples actividades en las que puede estar envuelto el ser humano, se han clasificado en básicas, genéricas, específicas y claves (Tobón, 2005). No se pretende aquí explicar cada una de ellas, pero si aclarar que, dependiendo de la actividad que desarrolle una persona, una competencia puede ser clasificada diferente.

3 Educación basada en competencias 
Como parte de los aportes y colaboraciones brindadas por la Unión Europea a Latino América, entre el 2004 y 2007 se desarrolló la versión latinoamericana de Proyecto Tuning, llevado a cabo previamente en Europa. Uno de sus cuatro ejes de trabajo era la definición de competencias por áreas temáticas. Producto de esta labor se establecieron las siguientes competencias específicas para el área de la Química (Beneitone, Esquetini, González, Maletá, Siufi, y Wagenaar, 2007, p. 274).

- Capacidad para aplicar conocimiento y comprensión en Química a la solución de problemas cualitativos y cuantitativos.

- Comprender conceptos, principios y teorías fundamentales del área de la Química.

- Interpretar y evaluar datos derivados de observaciones y mediciones relacionándolos con la teoría.

- Capacidad para reconocer y analizar problemas y planificar estrategias para su solución.

- Habilidad para desarrollar, utilizar y aplicar técnicas analíticas.

- Conocimiento y comprensión en profundidad de un área específica de la Química.

- Conocimiento de las fronteras de la investigación y desarrollo en Química.

- Conocimiento del inglés para leer, escribir y exponer documentos, así como comunicarse con otros especialistas.

- Capacidad para la planificación, el diseño y la ejecución de proyectos de investigación.

- Habilidad en el uso de las técnicas modernas de la informática y comunicación aplicadas a la Química.

- Habilidad para participar en equipos de trabajo inter y transdisciplinarios relacionados con la Química.

- Dominio de la terminología Química, nomenclatura, convenciones y unidades.

- Conocimiento de las principales rutas sintéticas en Química.

- Conocimiento de otras disciplinas científicas que permitan la comprensión de la Química.

- Habilidad para la presentación de información científica ante diferentes audiencias tanto en forma oral como escrita.

- Habilidades en el seguimiento a través de la medida y observación de propiedades químicas, eventos o cambios y su recopilación y documentación de forma sistemática y fiable.

- Dominio de las buenas prácticas de laboratorio. 
- Capacidad de actuar con curiosidad, iniciativa y emprendimiento.

- Conocimiento, aplicación y asesoramiento sobre el marco legal en el ámbito de la Química.

- Habilidad para aplicar los conocimientos de la Química en el desarrollo sostenible.

- Comprensión de la epistemología de la ciencia.

Adicionales a estas competencias específicas, el profesional en Química (al igual que muchos otros profesionales) debe de contar con capacidades más genéricas que ayudan a desempeñarse de la mejor manera. En relación a lo que conlleva una competencia, el Dr. Ribes Iñesta (2006) señala:

Las competencias implican habilidades, pero no constituyen solamente habilidades. Las competencias requieren, además, satisfacer un nivel o criterio de aptitud. Ser competente incluye ser hábil y ser apto. Apto proviene del latín aptus, que significa bien dispuesto, entrelazado, unido, ligado. El concepto de aptitud no se relaciona con conocimiento previo específico, sino con la propensión a actuar de cierta manera ligado a un criterio o requerimiento. Ser apto significa poder ajustarse de una manera y no de otra a una situación, de acuerdo a los criterios funcionales opcionales y a los resultados, logros o actos previstos por dichos criterios. Demostrar aptitud implica el ejercicio de ciertas habilidades y no de otras. (p. 21)

El desarrollo de una competencia va más allá de adquirir conocimiento, involucra un pensamiento crítico y un desempeño en contexto. Hay fuertes señalamientos a la poca relación entre lo que se enseña, cómo se enseña y su pertinencia profesional. Esto es sintetizado por Zurita (2000, p. 21) de la siguiente forma:

- Una formación profesional disfuncional a las necesidades que plantea la realidad.

- Rápida obsolescencia de lo aprendido en la fase de formación.

- Inseguridad en el trabajo y alta movilidad laboral.

- Autoreferencia y autocomplacencia de las universidades.

- La educación como la más alta prioridad de la universidad.

- Ausencia de una formación axiológica intencionada.

Si se analiza desde la perspectiva de que una competencia encierra el saber conocer, hacer y ser, la actual formación de los químicos y químicas en la Universidad de Costa Rica 
apenas está empezando a trabajar en el segundo apartado. Hay que tener presente que son las competencias genéricas las que complementarán a las específicas y permitirán a los profesionales adaptarse en el ambiente laboral (de los Ríos, Herrera, Letelier, Poblete y Zúñiga, 2000).

Es preciso tener presente que el mercado laboral es el que establece que características debe poseer el profesional, pues las posibilidades laborales son una pregunta presente en cualquier estudiante (Chavoya Peña y Reynaga Obregón, 2011). No hay que olvidar que la educación como función social debe responder a las necesidades de la sociedad y producir las transformaciones necesarias. Esto involucra que la universidad no solo transmita conocimiento, sino que promueva una formación integral, que facilite la formación a largo plazo y el paso del sistema educativo al mercado laboral (Escorcia, Gutierrez y Henríquez, 2007). Al respecto, Camarena Gómez y Velarde Hernández (2009) mencionan lo siguiente:

(...) por una parte, que las instituciones no incluyen la formación de conocimientos y capacidades que el desarrollo integral del país requiere; y, por la otra, que tampoco logran ajustarse a lo que el mercado empresarial demanda -conocimientos y capacidades técnicas flexibles a los requerimientos de la empresa-. (p. 111)

Las nuevas personas profesionales sufren al enfrentarse a un trabajo por el encuentro con un mundo desconocido, con costumbres propias, distinto vocabulario y valores; además, sufren por tener una formación muy teórica en relación a la aplicación en el trabajo (Parent Jacquening, Esquivel Estrada y Heras Gómez, 2004).

Como parte de las soluciones que las universidades han implementado para hacer más pertinente la formación profesional y para desarrollar competencias más alineadas con la realidad laboral, algunas de las carreras universitarias han ido incorporando un periodo de práctica supervisada o práctica profesional en contextos reales de trabajo, diferente a la universidad (Zabalza Beraza, 2011). Las carreras relacionadas con la educación y las de ciencias de la salud han sido pioneras en este tipo de prácticas. Tienen una estructura bastante definida y con objetivos de aprendizaje más claros.

La importancia que se le da a este tipo de cursos radica en la posibilidad que le brindan al estudiantado de construir y adquirir conocimiento profesional en un espacio donde la universidad y el sistema productivo convergen y contribuyen a la formación, por medio del 
desarrollo en los futuros y las futuras profesionales, de habilidades y actitudes características de un desempeño profesional (Morales Calvo, 2013).

Autores como Pañellas Valls y Palau Sanz (2012) argumentan que es una forma progresiva de que el estudiantado sean inmerso en la profesión. Según Delgado (2012), es preciso entender que la práctica profesional se compone de elementos que dinamizan, le dan sentido práctico y configuran la formación que se le brinda al alumnado universitario. García, Navío y Carson (citados por Armengol Asparó, Castro Ceacero, Jariot García, Massot Verdú y Sala Roca, 2011) definen la práctica profesional de la siguiente manera:

Existe un cierto consenso en definir el Practicum ${ }^{4}$ como una de las asignaturas más relevantes en los estudios de los futuros profesionales de la educación, ya que a lo largo de la asignatura de prácticas, el estudiante se enfrenta a situaciones complejas que no sólo requieren poner en funcionamiento los conocimientos y habilidades adquiridas en otras asignaturas de la titulación, sino que también tiene que poner en juego competencias que no se han adquirido en estas mismas asignaturas. (p. 73)

Sin embargo, existen críticas a los sistemas actuales de prácticas profesionales. Por ejemplo, se ha resaltado que el estudiantado cada vez está más expuesto a los problemas reales de su profesión, tratando de reducir la brecha entre saber conocer y saber hacer, pero se ha dejado de lado el desarrollo de un pensamiento crítico y la toma de decisiones. En otras palabras, la formación profesional se está tornando en un entrenamiento (Stewart, 2010).

Muchas de las dificultades también radican en que no existe una fundamentación clara de estas iniciativas, que conlleva una desvinculación del resto del plan de estudios y por ende una práctica académicamente rudimentaria con una supervisión generalmente remota (Zabalza Beraza, 2011).

En el marco de la práctica profesional en la carrera de Química de la Universidad de Costa Rica, surgen las siguientes interrogantes que motivan la presente investigación:

- ¿Cuáles son los conocimientos y habilidades más relevantes en el ejercicio profesional?

- ¿Qué aspectos son resaltables de la actual práctica profesional?

- ¿Qué conocimientos y habilidades se pueden reforzar?

4 Nombre que se emplea en España desde 1983 para designar a las antes denominadas prácticas. 


\section{Metodología}

En este apartado se describen los tres aspectos más importantes en el desarrollo de la presente investigación. En primera instancia se describe los sujetos que formaron parte de la recopilación de información; posteriormente, la metodología empleada para la elaboración y aplicación de encuestas y; finalmente, se realiza el análisis de los datos.

\subsection{Población consultada}

Para el presente estudio se elaboró una encuesta que se solicitó llenar a 101 graduados de Química que aprobaron el curso QU-0495 Industria e Investigación Química, que corresponde a casi la totalidad de los que finalizaron el curso entre del año 2010 a julio del año 2014. Este curso es el último en el plan de estudios de la carrera de Bachillerato en Química, por lo que se graduaron justo posterior a su finalización.

La investigación se restringió a las fechas antes citadas por no contarse con una base de datos con la información de los estudiantes graduados antes del 2010. Adicionalmente, la población consultada se graduó cumpliendo con el plan de estudios que entró en vigencia en el 2007.

\subsection{Elaboración y aplicación de la encuesta}

Las preguntas de la encuesta fueron elaboradas con la finalidad de responder las interrogantes que se plantean arriba. Para el diseño del cuestionario se empleó el método del "florero", el cual divide el cuestionario en una sección introductoria, preguntas que satisfagan los objetivos de información planteados y una sección de identificación para la caracterización de la población estudiada (Hair, Bush y Ortinau, 2009).

La formulación de las preguntas y las escalas de medición se elaboraron tomando en cuentas las sugerencias encontradas en la literatura (Hair et al., 2009; Trejos Zelaya y Moya Vargas, 2012). Después de redactarlas se solicitó a cinco profesores colegas docentes de la Escuela de Química y una profesional en orientación vocacional su revisión. Estas personas hicieron observaciones respecto a la redacción y las opciones de respuesta propuestas. Posteriormente, se validó con 4 graduados de la población sujeta a estudio, empleando el mismo mecanismo de aplicación que se describirá más adelante. Los sujetos encuestados identificaron problemas de forma en la plataforma electrónica utilizada, como no obligar la respuesta o no brindar múltiple opción de respuesta en alguna pregunta. Se dieron sugerencias en temas que consideraron como importantes de abarcar en la formación y 
necesario para el desempeño, como por ejemplo sistemas de gestión de calidad. Este proceso verificó que la comprensión de las preguntas fue acorde con el objetivo planteado en el momento de su construcción.

Una vez con el cuestionario validado, se procedió a hacerlo llegar a las personas graduadas a través de un enlace a la página "encuestafacil"5. Cabe señalar que dentro de las ventajas brindadas por el medio usado estuvo la posibilidad de modificar al azar la posición en que se presentan las opciones en preguntas donde se podía elegir entre varias o múltiples respuestas. El cuestionario fue enviado desde el correo electrónico institucional del investigador a las direcciones de correo electrónico registradas en la base de datos en el periodo 2010-2014. Desde el vínculo, quien sería encuestado o encuestada podía acceder a la ubicación de la encuesta sin necesidad de identificarse, por lo que todos los datos recogidos son totalmente anónimos. Es importante señalar que no se tenía certeza del número de personas que mantienen el mismo correo electrónico. Sin embargo, era de suponer que muchos lo utilizan aún, pues se trata de direcciones en los más populares proveedores gratuitos del servicio (gmail, hotmail y yahoo, principalmente).

\subsection{Análisis de los datos}

La información suministrada por quienes participaron en la encuesta fue manejada por el programa informático empleado por la plataforma en línea utilizada. Se obtuvo un reporte con los datos crudos. A partir de estos se comenzó a realizar las relaciones, procesar las estadísticamente y representarlas de la manera que se consideró más conveniente para su presentación.

Se realizó un análisis estadístico primordialmente descriptivo. Se trató de identificar tendencias centrales y de obtener frecuencias de respuestas para valorar la repetición de las mismas. En algunos casos los encuestados debían priorizar las respuestas con opciones múltiples, por lo cual se tiene una media de posición de elección.

\section{Resultados y análisis}

A continuación se exponen y discuten los resultados obtenidos, tratando de dar respuesta a las inquietudes que motivaron la investigación. Primero se describirán los hallazgos respecto a las características de la población, seguido por su percepción de la pertinencia de la formación y el periodo de práctica profesional.

5 www.encuestafacil.com 


\subsection{Características relevantes de la población estudiada}

Como se mencionó en la parte metodológica, la encuesta fue enviada a 101 personas graduadas, de las cuales $48(47,5 \%)$ la llenaron. De esas personas 25 fueron mujeres y 23 hombres, lo cual llama la atención y es consistente con un estudio de seguimiento laboral realizado por el Consejo Nacional de Rectores (CONARE). En dicho estudio se encuestó a los egresados y las egresadas de las universidades de Costa Rica entre el 2008 y 2010 empleando un muestreo estadístico al azar, que mostró una relación de (45:55) \% de hombres a mujeres en el caso específico de la carrera de Química (Gutiérrez Coto, Kikut Valverde, Navarro Cerdas, Azofeifa Ureña y Rodríguez Ramos, 2015). Tradicionalmente la carrera de Química fue mayoritariamente seleccionada por varones; sin embargo, los datos demuestran que el comportamiento se está invirtiendo.

Dos terceras partes de quienes completaron la encuesta se encuentran laborando en la actualidad. El tercio restante aún continúa estudiando. Por tanto, los resultados que se presentarán más adelante tendrán una mezcla de lo que se considera necesario para un desempeño profesional adecuado en la academia y fuera de esta.

En la Figura 1 se muestra cómo la mitad de las personas graduadas que participaron en la encuesta y trabajan lo hace en el sector privado, pero también una cantidad importante lo hace en el sector público. Esta información es relevante para interpretar las respuestas a algunas preguntas. Los conocimientos y habilidades requeridas para desempeñarse en cada sector pueden diferir significativamente.

Figura 1

Distribución por tipo de sector en que las personas encuestadas desempeñan sus trabajos

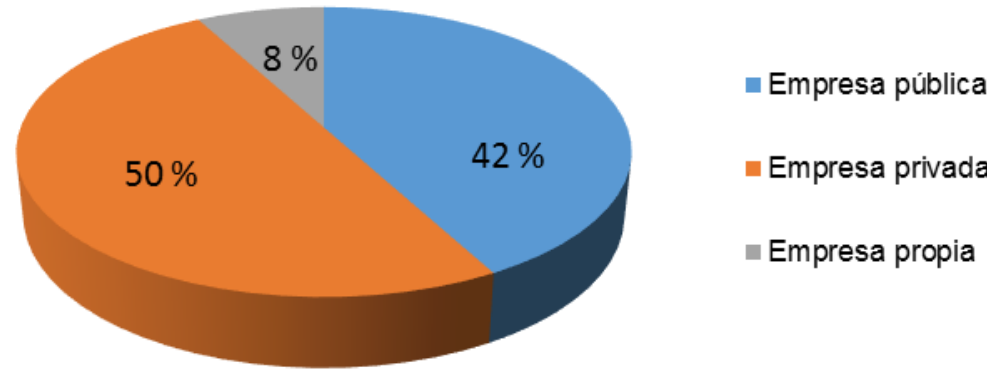

Fuente: Elaboración propia, 2015. 
El $88 \%$ de personas encuestadas realizan labores que están mucho o totalmente relacionadas con el campo de la Química. Además, un $61 \%$ tiene un salario inferior a los 750000 colones; un $31 \%$ entre 750000 y 1000000 de colones y el resto tiene un salario superior. Como último dato relevante en la caracterización de la población que contestó la encuesta, es que la duración promedio para concluir el bachillerato es de 5 años.

En términos generales es una población típica de estudiantes que se ha graduado recientemente. La relación de formación base con su actividad está muy relacionada, son pocos los que están ejerciendo labores diferentes a las de la Química. Esto también coincide con la distribución del ingreso. Es de esperar que entre más experiencia profesional tengan, mayor será su salario. Al ser egresados y egresadas que a lo sumo tienen 4 años de haberse graduado sus ingresos son relativamente bajos.

\subsection{Evaluación de la pertinencia de la formación}

Uno de los objetivos primordiales era recoger la percepción de las personas encuestas respecto a la relevancia de la formación que recibieron para su desempeño laboral. La Figura 2 muestra la proporción en que las personas mencionaron cada una de los grandes bloques en que se divide la formación en Química y su importancia en el desarrollo una vez graduadas.

Figura 2

Porcentaje de graduados que mencionaron como útil profesionalmente cada grupo temático dentro de la formación

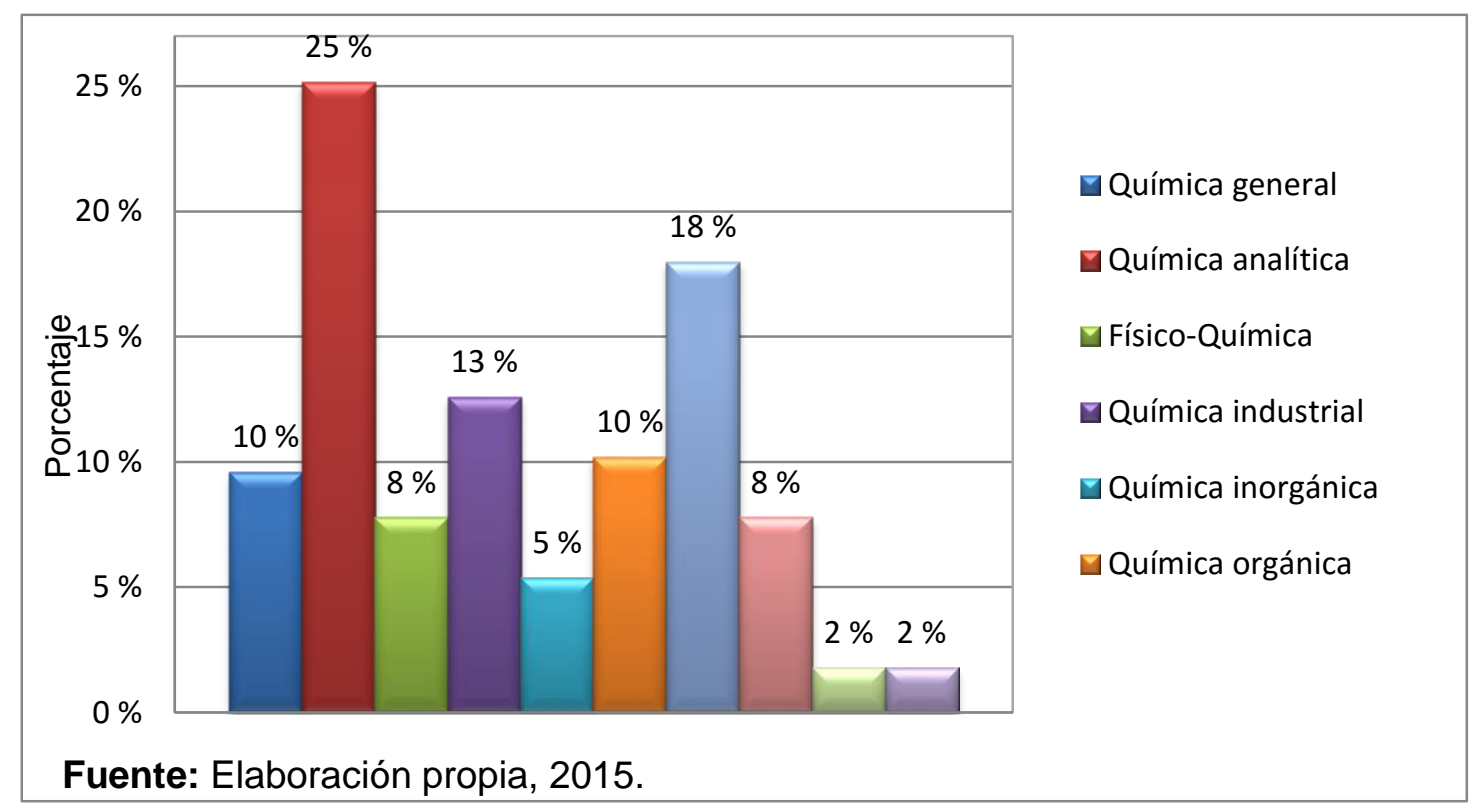


Las habilidades y conceptos más importantes son los relativos a la Química analítica, lo que no es de extrañar pues la labor profesional en el país gira mucho en torno a los análisis químicos y venta de instrumentación para los mismos. En segundo lugar, aparecen los cursos optativos relacionados con Química. Se puede concluir que el estudiante al tener la opción de gestionar su formación lo está haciendo pensando en sus preferencias profesionales. Por eso, los cursos que elige le son útiles en su trabajo.

Un poco más rezagado se encuentra la formación concerniente a la parte industrial. En alguna medida estos cursos están siendo capaces de transmitir conceptos que son necesarios en el trabajo. Sin embargo, si se considera que el $50 \%$ trabaja directamente en la empresa privada (que mayoritariamente tiene procesos industriales), el porcentaje de mención resulta muy bajo.

Llama la atención la poca importancia que se le brindó a la matemática y la física. Podría ser producto de que las herramientas adquiridas en estos cursos se emplean indirectamente por medio de la aplicación de los conceptos químicos. Para concluir, sobre esto habría que estudiar más detalladamente las funciones de cada persona encuestada.

Tratando de valorar la importancia de lo enseñado y aprendido por los químicos y las químicas encuestados, se consultó respecto a su opinión sobre algunos puntos que forman parte de currículo de la carrera y otros que deben desarrollar por su cuenta los estudiantes. El Cuadro 2 muestra la percepción de las personas encuestadas al respecto. Aquí, los primeros tres aspectos no son formalmente desarrollados en la carrera, mientras que los otros tres sí.

\section{Cuadro 2}

Número de encuestados que valoraron la importancia profesional de los aspectos consultados.

\begin{tabular}{|c|c|c|c|c|c|}
\hline $\begin{array}{l}\text { Objetivos de la } \\
\text { malla curricular }\end{array}$ & Aspecto & \multicolumn{4}{|c|}{$\begin{array}{l}\text { Valoración } \\
\text { Muy importante Importante Poco importante Irrelevante }\end{array}$} \\
\hline \multirow{3}{*}{ No } & Relaciones interpersonales & 34 & 13 & 0 & 1 \\
\hline & Dominio del idioma inglés & 33 & 13 & 1 & 1 \\
\hline & Conocimiento en temas diferentes a la química & 26 & 20 & 2 & 0 \\
\hline \multirow{3}{*}{$\mathrm{Si}$} & Conocimiento de los conceptos teóricos de química & 22 & 24 & 2 & 0 \\
\hline & Habilidades prácticas en química & 32 & 14 & 2 & 0 \\
\hline & $\begin{array}{l}\text { Conocimiento de los aspectos prácticos del } \\
\text { desarrollo profesional del químico(a) }\end{array}$ & 23 & 18 & 5 & 2 \\
\hline
\end{tabular}

Fuente: Elaboración propia, 2015. 
Se denota que conocimientos y habilidades que no se encuentran como objetivos de formación son evaluados como importantes para desempeñarse profesionalmente. Como se puede apreciar, son aspectos muy generales y no exclusivos de una carrera. Por otra parte, lo referente a las herramientas adquiridas y desarrolladas durante los años de universidad se muestra igualmente necesario. Aunque estas últimas fueron catalogadas en promedio como un poco menos importantes.

Se manifiesta como necesario dar énfasis e integrar a la formación en Química aspectos que no necesariamente tengan relación directa con la ciencia pura. No se debe dejar como responsabilidad del estudiantado desarrollarlos por cuenta propia, sino que se le debe apoyar, ya sea incorporando dichos aspectos como ejes transversales o como cursos específicos que ayuden en esto.

Al evaluar la pertinencia de la formación recibida es fundamental incorporar lo que realiza la Sección de Química Industrial, la cual es la encargada de contextualizar el conocimiento previo a la realidad nacional de la profesión. Con este objetivo se cuestionó a las personas encuestadas sobre la utilidad de los cursos brindados por la Sección en las labores que efectúan. La Figura 3 muestra la distribución de la valoración externada.

Figura 3

Percepción de las personas encuestadas respecto a la importancia de los cursos de la Sección de Industrial en el trabajo actual

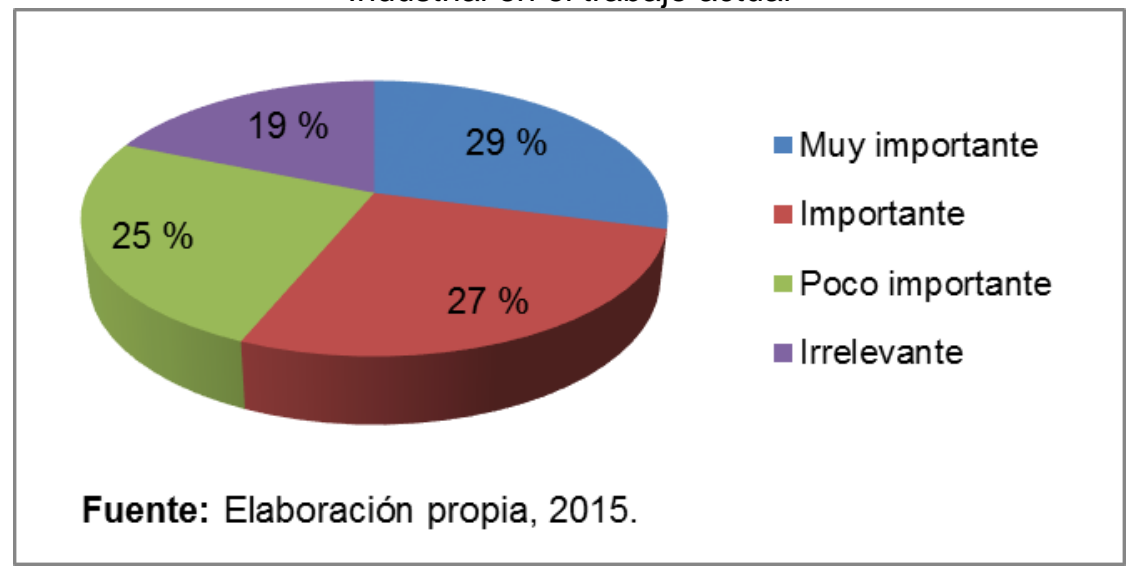

Existe una distribución muy similar entre las cuatro respuestas posibles. En apariencia la Sección debe mejorar su labor. Puede ser que exista un desconocimiento de las necesidades reales del mercado laboral o que falte un mayor esfuerzo en lograr una formación dual, con apoyo de las empresas e instituciones que emplean profesionales en Química. Esto se refuerza con la respuesta a la pregunta si estos cursos dan un panorama 
claro de la realidad de la industria Química en el país, a lo cual el $67 \%$ contestó negativamente.

Parece haber una discrepancia entre lo que se está enseñando en la carrera y lo que se necesita para el ejercicio profesional. Existen elementos que son ineludibles en la formación Química. No se puede olvidar que esta se cataloga como una ciencia básica o exacta, por lo que tiene conceptos fundamentales que tienen que ser aprendidos. Sin embargo, se debe reforzar la contextualización del conocimiento y el desarrollo de competencias genéricas y algunas específicas vitales para la incorporación al mercado laboral y el buen desempeño.

En el desarrollo curricular básicamente existen dos enfoques: el que se podría denominar clásico con cuatro ejes como son la docencia, investigación, acción social e internacionalización, y el que considera la formación por medio de una serie de reglas y prácticas que lleven al estudiante al desarrollo de competencias óptimas para su desempeño (Escorcia et al., 2007).

La carrera de Química sigue estancada en el modelo clásico, pero se puede percibir que esta estructuración no está dando los mejores frutos por lo presentado anteriormente. En este punto queda repensar la conveniencia del modelo y decidir sobre las adaptaciones requeridas para lograr formar el tipo de profesionales en Química que la sociedad costarricense demanda.

\subsection{Evaluación de la práctica profesional}

Uno de los puntos importantes a evaluar fue la utilidad del curso Industria e Investigación Química (práctica profesional) en el desempeño de los estudiantes una vez graduados. Este es el último requisito académico para optar por el título de Bachillerato en Química y busca acercar a la futura persona profesional a la realidad laboral que enfrentará.

En primera instancia, se puede rescatar que alrededor de la mitad no aprendieron conceptos químicos nuevos en su práctica profesional. Esto no es de extrañar porque la mayor parte de la industria Química costarricense se fundamenta en principios básicos de la Química, conocidos y estudiados desde hace muchos años. Son pocas las empresas o instituciones que están a la vanguardia tecnológica y que puedan aportar información nueva o de punta a quien será profesional (ver Cuadro 3).

Al parecer, tampoco aporta a la colocación laboral de la futura graduada o el futuro graduado. El $23 \%$ manifiesta que le ayudó a conseguir trabajo, dato que concuerda con la 
información en la base de datos del curso; y el $25 \%$ ha manifestado que al finalizar su práctica se ha quedado laborando en el lugar en el cual la hizo. Hay que tomar en consideración que la mayoría de empresas o instituciones son medianas o grandes, con una rotación de personal baja y un crecimiento lento. Es difícil que en estas condiciones se den contrataciones frecuentes de profesionales en Química. Cerca de la mitad de las personas encuestadas señala haber establecido contactos importantes durante su práctica; dicha proporción se puede considerar baja si se vislumbra que es la primera experiencia laboral que tiene y que debería ayudar de forma categórica en su futuro profesional.

\section{Cuadro 3}

Valoración de afirmaciones referentes a los logros de la práctica profesional.

\begin{tabular}{|c|c|c|c|c|c|}
\hline Afirmación & $\begin{array}{l}\text { Muy de } \\
\text { acuerdo }\end{array}$ & $\begin{array}{c}\text { De } \\
\text { acuerdo }\end{array}$ & $\begin{array}{c}\text { Valoración } \\
\text { Ni de acuerdo ni } \\
\text { desacuerdo }\end{array}$ & Desacuerdo & $\begin{array}{c}\text { Muy } \\
\text { desacuerdo }\end{array}$ \\
\hline Aprendí conceptos químicos nuevos & $15 \%$ & $31 \%$ & $25 \%$ & $15 \%$ & $15 \%$ \\
\hline Me ayudó a conseguir trabajo & $17 \%$ & $6 \%$ & $40 \%$ & $21 \%$ & $17 \%$ \\
\hline Conocí la realidad profesional química & $29 \%$ & $35 \%$ & $27 \%$ & $2 \%$ & $6 \%$ \\
\hline $\begin{array}{l}\text { Identifiqué aspectos a mejorar como } \\
\text { futuro(a) profesional }\end{array}$ & $33 \%$ & $38 \%$ & $21 \%$ & $2 \%$ & $6 \%$ \\
\hline Hice buenos contactos & $15 \%$ & $31 \%$ & $29 \%$ & $13 \%$ & $13 \%$ \\
\hline $\begin{array}{l}\text { Aprendí conceptos nuevos no } \\
\text { relacionados con química }\end{array}$ & $31 \%$ & $46 \%$ & $13 \%$ & $8 \%$ & $2 \%$ \\
\hline Me ayudó a establecer mi plan de vida & $13 \%$ & $19 \%$ & $33 \%$ & $17 \%$ & $19 \%$ \\
\hline Tuve poco aprendizaje & $10 \%$ & $8 \%$ & $21 \%$ & $29 \%$ & $31 \%$ \\
\hline El curso me resultó molesto & $2 \%$ & $6 \%$ & $25 \%$ & $29 \%$ & $38 \%$ \\
\hline $\begin{array}{l}\text { Mi visión de la profesión química varió } \\
\text { de antes a después de la práctica }\end{array}$ & $27 \%$ & $35 \%$ & $27 \%$ & $6 \%$ & $4 \%$ \\
\hline
\end{tabular}

Fuente: Elaboración propia, 2015.

Por otra parte, es importante resaltar que a pesar de que se trata de un curso muy diferente dentro de la malla curricular de la carrera, y para el cual los estudiantes no han sido preparados formalmente, el curso no les resulta molesto. También se cumple otro objetivo fundamental: que los futuros y las futuras profesionales aprendan conceptos nuevos no relacionados con la Química pero que son vitales para el desempeño profesional. Además, existe un importante porcentaje de personas graduadas que manifestó que su visión de la profesión Química en el transcurso de la práctica profesional varió. Esto deja entrever que la formación de los primeros años no está brindando una perspectiva clara de la labor que 
realizan los profesionales en Química. Podría ser una falta de alineamiento entre las necesidades de la sociedad y el enfoque de la universidad.

Dentro de los tópicos señalados como importantes de cubrir durante la formación, resalta el de emprendimiento e innovación. En apariencia es un vacío que no es abarcado por el currículo de la carrera. Hay que resaltar que no es una minoría la que menciona esto.

\subsection{Consecuencias sobre el diseño curricular de la carrera}

Es claro que las expectativas de los estudiantes y de la sociedad han ido variando con el tiempo. Las condiciones de oferta académica se han ampliado, tanto en la propia Universidad de Costa Rica, con la apertura del Bachillerato y Licenciatura en Laboratorista Químico, como por la Universidad Nacional, con la carrera de Química Industrial. Adicionalmente, en los últimos meses la Universidad Latinoamericana de Ciencia y Tecnología anunció la oferta de la carrera de Licenciatura en Ingeniería Química Industrial (Universidad Latinoamericana de Ciencia y Tecnología, 2015).

Sin duda esto ha ejercido una mayor competencia por los campos laborales, aunque en la actualidad no existen problemas graves de desempleo. Los datos publicados por el Observatorio Laboral de Profesiones respecto al seguimiento laboral de quienes se graduaron entre los años 2008-2010, muestra una desocupación del 4,52 \% para profesionales en Química (provenientes de los programas de la Universidad Nacional y Universidad de Costa Rica), aproximadamente la mitad del desempleo general del país.

Parece ser que la formación que se brinda actualmente no es del todo útil en el desempaño profesional. Solo los conocimientos relacionados con Química analítica son mencionados por un cuarto de las personas encuestadas como relevantes para su desarrollo profesional (ver Figura 2). Resulta alarmante que los cursos de Química industrial, que deberían ser útiles para su desempeño laboral, en apariencia no lo son tanto.

Cada vez es más cierto que los conocimientos no son lo más importante para obtener un puesto de trabajo; por el contrario, el peso se está inclinando a otras características, es decir: "muchas empresas al contratar profesionales jóvenes no están buscando determinados conocimientos, sino personas con las competencias que ellas requieren" (Cordua, 2000).

Dentro de este cada vez más complejo panorama, existen varias tendencias que tratan de guiar el perfil profesional de las personas egresadas. Las más importantes a saber: la empresarial, la pública, la socio-cultural y educativa. No hay que dejar de lado la influencia 
indirecta de lo que se ha llamado globalización. Cada día más empresas son transnacionales, se produce más movilidad laboral, los gobiernos firman acuerdos internacionales y la sociedad se ve influenciada por las tendencias globales de moda.

Cada uno de estos actores -representados por personas, organizaciones 0 instituciones- juega un papel importante dentro de los objetivos particulares que en conjunto orientan las necesidades de formación de los futuros y las futuras profesionales y que están acordes con los requerimientos para el desarrollo del país.

Sin embargo, con los datos mostrados anteriormente se percibe una discrepancia entre lo que la Universidad de Costa Rica ofrece como perfil de salida de personas egresadas en Química y lo que el mercado laboral está requiriendo. Aspectos que no son abarcados dentro el programa de estudio son valorados como igual o más importantes que los que sí se incluyen (ver Cuadro 2). Esto no significa que se deba eliminar de la formación cierto conocimiento o desarrollo de habilidad, lo que sí deja de manifiesto es que se debe procurar un equilibrio entre lo que ya se hace bien y lo que está pendiente por incorporar y hacer.

Mucho se ha hablado de las competencias profesionales, y en el presente documento se han citado algunas definidas para un profesional en Química. Pero la formación no solo se trata de esto, también involucra otros objetivos educativos que pueden estar explícitos o no, pero deben ser comprobables en el egresado. La síntesis de los objetivos fundamentales que plantean de los Ríos et al. (2000) se brinda a continuación:

- La adquisición de conocimiento: son importantes los conocimientos referenciales de la disciplina y que los egresados "conozcan" el contexto social en que se desenvolverán. Esto les facilitará el desarrollo de un pensamiento crítico y capacidad de aprendizaje en el futuro.

- El cultivo de habilidades: se debe facilitar el cultivo de habilidades esenciales como memorizar, clasificar e inferir que le servirán de base al egresado para el desarrollo de habilidades específicas.

- El cultivo de actitudes y valores: Las formas de actuar o de responder en determinadas situaciones son soportadas en actitudes como comportamiento ético, la responsabilidad, el pensamiento crítico, entre otros.

- El desarrollo de destrezas: habilidades propias que se requieren para el buen desempeño de una actividad. Habitualmente están asociadas al uso de equipo e instrumentación en el caso de la Química. 
Una competencia profesional encierra los objetivos anteriores y va más allá. Es una mezcla de saber conocer, saber hacer y saber ser en un contexto determinado. Resulta prácticamente imposible para las universidades representar todos los posibles contextos en que se puede desenvolver quien se egresa, pero si debe generar condiciones adecuadas para que el estudiante desarrolle competencias generales y específicas útiles en la mayor parte de situaciones laborales.

Como ya se expuso anteriormente, las prácticas profesionales se han planteado como uno de los mecanismos para tratar de acortar esta brecha entre los objetivos académicos o educativos y los profesionales o empresariales. Sin embargo, esta actividad por sí misma no asegura nada respecto a la idónea formación del futuro o la futura profesional. En este sentido, es pertinente resaltar lo que dice Zabalza Beraza al respecto:

(...) Cuando se entiende que el Practicum es enviar a los estudiantes a centros de trabajo, las instituciones suelen contentarse con que ese propósito se cumpla y tienden a medir su éxito por el número de estudiantes que salen de prácticas o por el número de centros de prácticas vinculados al programa. En tales casos, parece obvio que los problemas que deben resolverse son, básicamente, de orden organizativo. Pero el problema aparece cuando las instituciones se detienen en ese nivel de desarrollo del Practicum, quizás porque carecen de referentes, de planes estratégicos o de voluntad institucional para proponerse objetivos más avanzados. (2011, p. 31)

En la Escuela de Química la práctica profesional todavía se encuentra en este nivel más organizativo. Las valoraciones aún son superficiales (ver Cuadro 3), pues no existe un fuerte ligamen entre las empresas o instituciones y la Escuela. Es cierto que los responsables en los centros de trabajo no son académicos, pero no se ha dado el espacio para que estas personas colaboren en una evaluación objetiva y más cercana a las necesidades académicas (Zabalza Beraza, 2011).

Tampoco existe una estructuración clara de los objetivos de aprendizaje que se desean alcanzar, lo que aun dificulta más la evaluación. La informalidad de la relación universidad y centro de trabajo hace que no se tenga la facilidad de establecer objetivos acordes con los requerimientos del estudiantado y del lugar donde se hace la práctica. Esto lleva a que no se dé un seguimiento adecuado, ni la tutoría idónea para que todo lo vivido no quede solo como bonitas experiencias (Zabalza Beraza, 2011). El acompañamiento queda a la libre y es poco homogéneo, resultando en estudiantes que reciben una excelente retroalimentación y otros, una muy pobre. 
Lo anterior se une a la falta de entrelazamiento de la malla curricular y lo que se requiere para la práctica profesional. La misma se convierte en un curso más, desligado de los cursos que deben brindar los insumos para el desarrollo de competencias profesionales. Esto sin mencionar los temas que no son abarcados en la carrera y son importantes profesionalmente (ver Figura 4). Muchos de estos puntos podrían ser cubiertos con el diseño de objetivos específicos a cumplir durante el desarrollo de la práctica, establecidos después de un acuerdo entre los objetivos del centro que recibe al estudiantado, la Escuela y el estudiante. Aquí, es importante citar a Zabalza Beraza (2011), quien menciona: "como decía el dicho Masai, «para educar a un niño, hace falta toda la tribu», también podemos reconocer que para formar a un buen profesional se precisa de la colaboración de toda la sociedad" (p.23).

\section{Figura 4}

Número de personas graduadas que consideran cada tema consultado como importante de abarcar en la formación y necesario para el desempeño profesional

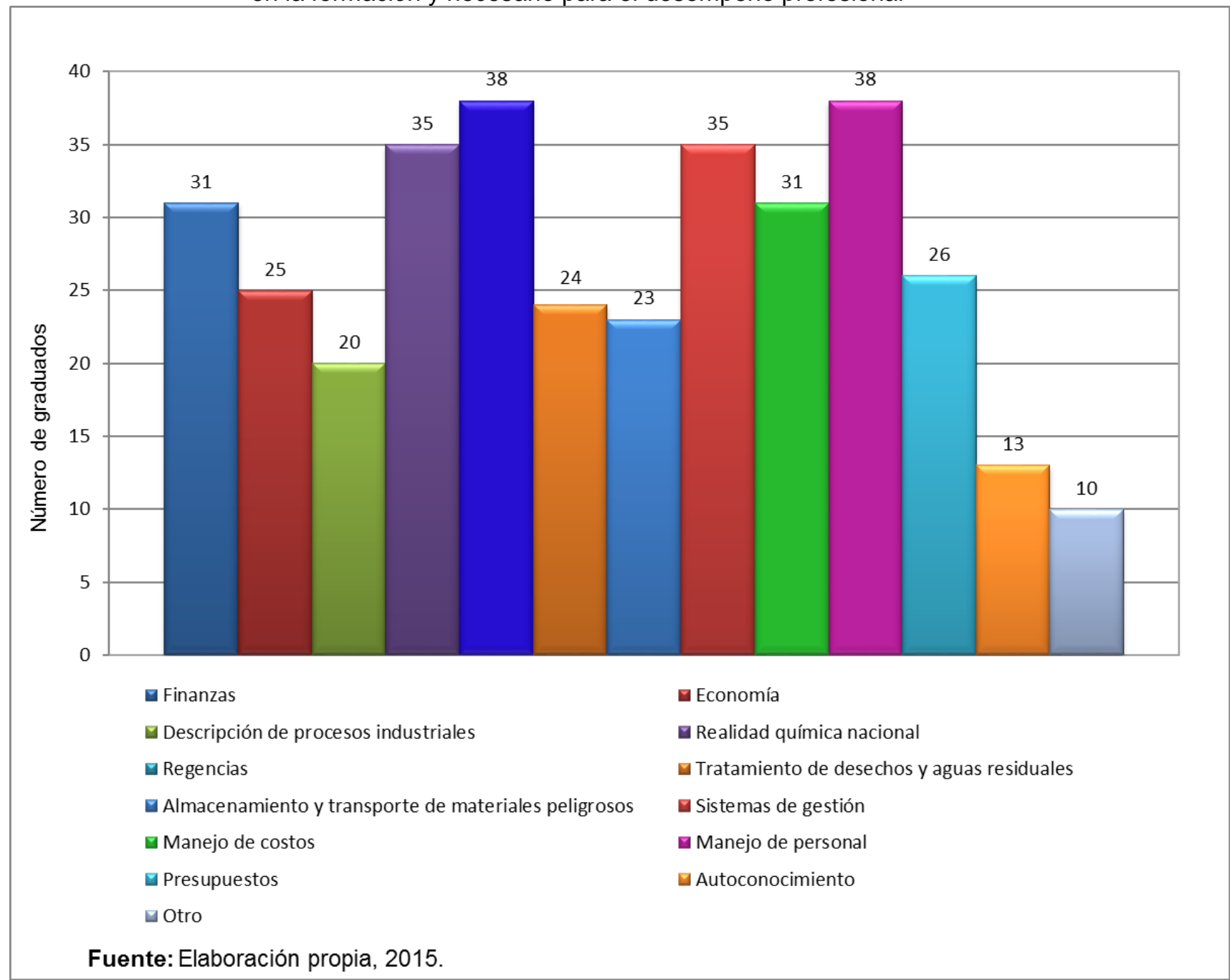


Se evidencia un problema entre el perfil profesional del químico que requiere la sociedad y el perfil de la persona egresada de la carrera en Química. En la actualidad la competitividad es un aspecto fundamental. Es preciso buscar los mecanismos para lograr formar profesionales en Química más acordes con lo que requiere la sociedad, sin dejar de lado aspectos académicos que siguen siendo esenciales para una persona profesional en Química.

\section{Conclusiones y recomendaciones}

Según la percepción de los egresados y las egresadas en el periodo bajo estudio, existe un distanciamiento entre lo que se enseña en la carrera de Química y lo que el futuro trabajador necesita. Los bloques temáticos no parecen estar aportando directamente al desarrollo profesional de quien se gradúa (con excepción de la Química analítica).

Lo más preocupante es que los cursos orientados al componente industrial son percibidos como uno de estos bloques de poca ayuda y que no dan un panorama claro del papel del profesional en Química en la realidad de la sociedad costarricense.

Es preciso reforzar áreas que no están relacionadas directamente con la parte dura de la ciencia Química, sino con las denominadas habilidades blandas. No se discute que en una ciencia básica se brinde prioridad a los conocimientos, pero hay que dejar espacios para la creatividad, innovación y el trabajo interdisciplinario.

Con esta valoración y considerando que la Escuela de Química ha iniciado el proceso de revisión del plan de estudios, se recomienda estructurar una malla curricular con base en el aporte de las personas egresadas y empleadoras, de tal forma que se adapte más a la realidad del país. Es indispensable que el currículum sea más versátil, con la posibilidad de que el estudiantado escoja una parte significativa de los temas que desea conocer, a través de cursos optativos. O bien, abrir énfasis que sean necesarios para el desarrollo del país y le den al estudiantado la opción de enfocarse en lo que encuentra más afín a sus preferencias y habilidades.

En estos énfasis deben considerarse las nuevas tendencias en la instrumentación Química y las tecnologías desarrolladas a partir de la investigación. Por supuesto, tiene que darse un alineamiento entre estas tendencias, lo que se aplica en la industria nacional y aspectos que son globales con particularidades locales. En este último sentido, lo relativo al ambiente surge como preponderante, aunado a la problemática del agua y energía que se observa en la actualidad. 
Por otra parte, las habilidades blandas deben ser desarrolladas durante toda la carrera universitaria. Deben constituirse como ejes transversales en la malla curricular. El reforzamiento debería ser constate y contextualizado. También las estrategias y metodologías de enseñanza deben ser revisadas y modificadas, con el propósito de lograr la transposición didáctica. Una alternativa es efectuar un proceso de enseñanza-aprendizaje contextualizado y por medio de problemáticas reales. Es decir, a partir del enfrentamiento del estudiantado con una situación real, este incorpora conocimientos, desarrolla habilidades y actitudes que le facilitan el desarrollo de pensamiento crítico y las destrezas sociales; siempre con la mediación docente.

Es necesario tener una práctica profesional más acorde con las necesidades de formación del estudiantado. Debe ser un eje transversal durante toda la carrera que culmine con una actividad práctica en una empresa o institución, donde existan objetivos claros de aprendizaje y una verdadera formación dual que termine de reforzar las competencias profesionales que requiere un químico o una Química.

La responsabilidad de los formadores y las formadoras de futuros profesionales en Química es doble. Por una parte, deben darle las herramientas al estudiantado para que pueda surgir y junto a este Costa Rica; por otro lado, deben responder a una sociedad que brinda los recursos para invertir en estas personas jóvenes que deben sostener el Estado solidario.

\section{Referencias}

Armengol Asparó, Carmen, Castro Ceacero, Diego, Jariot García, Mercè, Massot Verdú, Margarita y Sala Roca, Josefina. (2011). El Practicum en el Espacio Europeo de Educación Superior (EEES): mapa de competencias. Revista de Educación, 354(1), 7198.

Beneitone, Pablo, Esquetini, César, González, Julia, Maletá, Maida, Siufi, Gabriela y Wagenaar, Robert. (2007). Reflexiones y perspectivas de la educación superior en América Latina. Informe Final-Proyecto Tuning-América Latina. [versión digital pdf]. Recuperado

de http://tuning.unideusto.org/tuningal/index.php?option=com_docman\&task=docclick\&ltem $\mathrm{id}=191$ \&bid $=54 \&$ limitstart $=0$ \&limit $=5$

Camarena Gómez, Beatriz, y Velarde Hernández, Delisahé. (2009). Educación superior y mercado laboral: vinculación y pertinencia social ¿Por qué? y ¿Para qué?. Estudios Sociales, 17, 105-125. Recuperado de http://www.scielo.org.mx/scielo.php?script=sci arttext\&pid=S0188-45572009000300005 
Chavoya Peña, María y Reynaga Obregón, Sonia. (2011). Las licenciaturas en Jalisco, México: crecimiento, diversificación y complejización de la educación superior y su impacto en la pertinencia. Espacio Abierto Cuaderno Venezolano de Sociología, 20(2), 289-311.

Cordua, Joaquín. (2000). La formación universitaria en la perspectiva del mundo empresarial. En Las nuevas demandas del desempeño profesional y sus implicancias para la docencia universitaria (pp. 107-120). Recuperado de http://www.cinda.cl/download/libros/LASNUE 1.PDF

De los Ríos, Danae, Herrera, José, Letelier, Mario, Poblete, Álvaro y Zúñiga, María. (2000). Paradigmas y competencias profesionales. En Las nuevas demandas del desempeño profesional y sus implicancias para la docencia universitaria (pp. 43-89). Recuperado de http://www.cinda.cl/download/libros/LASNUE 1.PDF

Delgado, René. (2012). La Práctica Profesional como Espacio de Aprendizaje. Una perspectiva desde el enfoque de eje de la formación inicial universitaria. Revista Universitaria de Investigación y Diálogo Académico, 8(2), 71-104.

Ministerios Europeos de Educación. (1999). Declaración de Bolonia. Recuperado de http://www.ond.vlaanderen.be/hogeronderwijs/bologna/links/language/1999 Bologna D eclaration Spanish.pdf

Escorcia, Rolando, Gutiérrez, Alex y Henríquez, Hermes. (2007). La educación superior frente a las tendencias sociales del contexto. Educación y Educadores, 10(1), 63-77.

Gutiérrez Coto, Ilse, Kikut Valverde, Lorena, Navarro Cerdas, Gustavo, Azofeifa Ureña, Cinthia y Rodríguez Ramos, Nancy. (2015). Seguimiento de la Condición Laboral de las Personas Graduadas 2008-2010 de las Universidades Costarricenses [versión digital pdf]. Recuperado de https://www.conare.ac.cr/servicios/category/33-observatorio-deprofesiones

Hair, Joseph, Bush, Robert y Ortinau, David. (2009). Investigación de Mercados (4a․ ed.). México, D.F.: McGraw Hill.

Irigoyen, Juan, Jiménez, Miriam y Acuña, Karla. (2011). Competencias y educación superior. Revista Mexicana de Investigación Educativa, 16(48), 243-266.

Morales Calvo, Sonia. (2013). Evaluando el practicum en Educación Social : acciones de mejora ante la puesta en práctica de los nuevos grados. Revista de Docencia Universitaria, 11(1), 349-364.

Pañellas Valls, Mercè y Palau Sans, Rosa. (2012). El Practicum en los grados en Educación Infantil y Primaria: una aproximación progresiva a la realidad profesional. Revista de Docencia Universitaria, 10(1), 369-388.

Parent Jacquening, Juan, Esquivel Estrada, Noé y Heras Gómez, Leticia. (febrero, 2004). La práctica profesional una función indispensable. Ponencia presentada en el Cuarto 
Congreso Nacional y Tercero Internacional "Retos y Expectativas de la Universidad", Coahuila, México.

Posada Álvarez, Rodolfo. (2001). Formación Superior Basada en Competencias, Interdisciplinariedad y Trabajo Autónomo del Estudiante. Revista Iberoamericana, 34, 134.

Ribes Iñesta, Emilio. (2006). Competencias conductuales: su pertinencia en la formación y práctica profesional del psicólogo. Revista Mexicana de Psicología, 23(1), 19-26.

Stewart, David. (2010). The Purpose of University Education. The Psychologist-Manager Journal, 13, 244-250.

Tobón, Sergio. (2005). Formación Basada en Competencias (2da ed.). Bogotá: Ecoe Ediciones.

Trejos Zelaya, Javier y Moya Vargas, Ericka. (2000). Introducción a la Estadística Descriptiva (2da ed.). San José: Universidad Latina.

Universidad Latinoamericana de Ciencia y Tecnología. (2015). Plan de estudios Licenciatura en Ingeniería Química Industrial. Recuperado de http://www.ulacit.ac.cr/files/planestudio/pg400-028.pdf

Zabalza Beraza, Miguel. (2011). El Practicum-en la formación universitaria: estado de la cuestión. Revista de Educación, 354, 21-43. Recuperado de http://www.revistaeducacion.mec.es/re354/re354 02.pdf

Zurita, Reginaldo. (2000). La crisis de confianza en el conocimiento profesional. Implicaciones para la docencia universitaria. En Las nuevas demandas del desempeño profesional y sus implicancias para la docencia universitaria (pp. 16-50). Recuperado de http://www.cinda.cl/download/libros/LASNUE 1.PDF 